\title{
$3 \| \begin{aligned} & \text { FUNDAMENTOS TEÓRICOS DEL } \\ & \text { DÍA ESCOLAR DE LA NO-VIOLENCIA }\end{aligned}$ Y LA PAZ» (DENIP) \\ Llorenç Vidal \\ Doctor en Filosofía y Letras \\ Fundador del DENIP
}

"La Veritat és Una, però les seves formes són infinites $i$ els seus noms són incomptables. Tu cerca la teva Llum interior $i$ segueix-la fins al final, perquè en aqueixa Llum interior hi trobaràs la teva forma personal de 1 Única, Infinita i Eterna Veritat".

Petit llibre d un solitari, 3

"La Verdad es Una, pero sus formas son infinitas $y$ sus nombres son incontables. Tú busca tu Luz in interior y síguela hasta el final, porque en esta Luz interior encontrarás tu forma personal de la Única,

Infinita y Eterna Verdad".

Pequeño libro de un solitario, 3

El Día Escolar de la No-violencia y la Paz (DENIP) fue fundado en 1965, "cuando ni en España ni en el extranjero existía iniciativa similar alguna, fue mantenido contra viento y marea en circunstancias muy difíciles y es considerado la más importante experiencia pionera de Educación Pacificadora de nuestro tiempo, así como un núcleo dinámico de promoción de la misma a nivel nacional e internacional» 1 .

1 Eulogio Díaz del Corral: Historia del pensamiento pacifista y no-violento contemporáneo, Edit. Hogar del Libro, Barcelona, 1987, p. 128. 
Sin embargo esta experiencia dinámica de Cultura y Educación en y para la No-violencia y la Paz, que está perviviendo y creciendo activamente y en constante difusión en centros educativos de todo el mundo ya por más de cuarenta años, como todas las cosas humanas tuvo su inicio y algún día tendrá su final. Conocedor de la evidencia de este hecho, escribí en «No-violencia y Escuela».:

"El Día Escolar de la No-violencia y la Paz-que en nuestra desquiciada sociedad actual tiene una misión eminentemente anunciadora, profética e idealista-práctica apoyada en la esperanza en la posibilidad de un mundo mejor-es, desde 1964, una antorcha viva en la cual, consciente o inconscientemente, han encendido y encienden su llama numerosas acciones, grupos y movimientos de Educación Pacificadora. No podemos predecir con exactitud lo que será de él en el futuro, al igual que en los inicios de los años sesenta nadie podía imaginar su nacimiento ni la positiva atmósfera educativa que, entorno al mismo se ha creado...

"Algunos pueden pensar que es difícil, que la fuerza del DENIP es mínima y que son muchos los obstáculos... Sin duda, el ideal es elevado, como elevados son todos los ideales que pueden mejorar de veras a la Humanidad; pero no olvidemos la parábola evangélica del grano de mostaza, que es la más pequeña de todas las semillas, pero, cuando ha crecido, resulta más alta que las hortalizas y se hace un árbol, de forma que las aves del cielo se posan en sus ramas ${ }^{2}$.

"Si, a pesar de su horizonte ilimitado, algún año, al llegar el 30 de enero, el Día Escolar de la No-violencia y la Paz-este pequeño destello de la Llama Universal-, después de haber cumplido total o parcialmente con su misión de promover una Educación Pacificadora de carácter universal, abierto, profundo y permanente, deja de iluminar nuestras escuelas, resuenen en nuestra memoria las palabras de Rabindranath Tagore en sus Alocuciones en Shanti-Niketan:

Ninguna llama arde eternamente. O el aceite se consume o el viento apaga su luz. Muchas veces, también, la lámpara se rompe.

Con frecuencia, en un arranque de rebeldía podemos decir que la fuerza de las tinieblas es la última y verdadera fuerza, o que nosotros mismos, porque encendemos lámparas, somos los creadores de la luz. Pero, en realidad, apagar una luz sirve para demostrar que la fuente de la luz es inagotable y que el verdadero poder de los hombres consiste en su aptitud para poderlo demostrar siempre nuevamente» $3 y$.

\footnotetext{
2 MAT., 13: 31-32.

3 RabindRanath Tagore: Alocuciones en Shanti-Niketan. Edit. Cervantes, Barcelona, 1928, pp. $139-140$.

${ }^{4}$ LlORENÇ VIDAL: No-violencia y Escuela. El >Día Escolar de la No-violencia y la Paz=como experiencia práctica de Educación Pacificadora. Edit. Escuela Española, Madrid, 1985, pp. 137-139.
} 
Si la Pedagogía surge de la reflexión sobre el hecho educativo, reflexión sobre la que se construye una teoría descriptiva y orientadora de la práctica educativa y que, en desarrollos especializados posteriores, se diversifica en el conjunto denominado Ciencias de la Educación, podemos preguntarnos, ¿cuál es la reflexión filosófica y pedagógica que sirve de apoyo, base y dinamismo del Día Escolar de la No-violencia y la Paz?

En 1964 y después de una gestación anterior, el DENIP brota de la reflexión pedagógica sobre la filosofía y la práctica del pensamiento y de la acción no-violenta gandhiana, entendida en su sentido primigenio y etimológico del ahimsa, es decir, como la actitud de renunciar a matar y a dañar a los demás seres por medio del pensamiento, la palabra y la acción. Comprendida así, es el ejercicio de lucha humanizado por el amor, que apela a la razón y actúa respetando la naturaleza, la vida y la libertad de los demás. Es respeto integral por la vida.

El Padre de la independencia de la India la llama satyagraha, que en sánscrito quiere decir "firmeza en la verdad", y nos habla así:

"La no-violencia es la fuerza de la verdad... es la fuerza del espiritu... es la fuerza del amorn 5 .

Por esto hemos de considerar que el punto básico de la concepción filosófica y educativa gandhiana debe ser centrado sobre los conceptos de ahimsa y de satyagraha, conceptos que en el pensamiento de Gandhi llegan a fundirse de tal modo que se convierten en las dos caras de una misma medalla, de manera que no pueden subsistir el uno sin el otro. Y hasta tal punto son inseparables que la expresión occidental no-violencia abarca, en la mayoría de los expositores, el doble sentido de firmeza en la verdad que define el satyagraha y acción sin violencia que define el ahimsa, sin olvidar que ahimsa es no-violencia en el sentido positivo de "identificación con todo lo que vive» 6 .

Y Lanza del Vasto, que la define como la «fuerza de la justicia», declara:

"El héroe es el defensor de la justicia mediante la fuerza.

«El héroe puro es el defensor de la justicia mediante la fuerza de la justicia.

«La fuerza de la justicia es la definición correcta de la no-violencia»7.

5 Mahatma Gandhi, cita compuesta recogida en Llorenç Vidal y Eulogio Diaz del CoRRal en Ideario No-violento. 33 preguntas y respuestas sobre la No-violencia y la Paz, Quaderns Literaris Ponent, Mallorca, 1981, p. 7.

6 Mahatma Gandhi: Historia de mis experiencias con la Verdad (Autobiografía), Edit. Kraft, Buenos Aires, 1956, p. 470.

7 LANZA DEL VASTO: Las cuatro plagas, Sur, Buenos Aires, 1961, p. 295. 
Pero (cuidado! esta fuerza de la verdad, del espíritu, del amor, de la justicia y de la razón, no es la fuerza bruta, violenta, guerrera y militar puesta al servicio de estos hipotéticos objetivos, sino la fuerza superior, pacífica, enérgica, razonable, constante -tozuda, incluso, diría yo- y sin violencia que emana de estos supremos valores.

En su dimensión social la no-violencia incluye las técnicas no-violentas de manifestación pacífica, de no-cooperación y de desobediencia civil. Sin embargo no debemos nunca olvidar que la no-violencia es fundamentalmente una actitud de vida, una forma de comportamiento personal e interpersonal, individual y colectivo, y que existen tantas formas de no-violencia como personas o grupos viven este ideal.

En el «Dhammapada».o «Libro de la Palabra de Budha».encontramos estos versículos:

"Todos los seres temen el dolor, todos los seres tienen miedo a la muerte. Considera a cada ser como a ti mismo: No atormentes, no mates...

"Todos los seres temen el dolor, todos los seres aman la vida. Considera a cada ser como a ti mismo: No atormentes, no mates»8.

El segundo pilar en el que descansa el Día Escolar de la No-violencia y la Paz es el concepto de paz positiva y de pacifismo no-violento integral. Generalmente tenemos una idea superficial de la paz, considerada negativamente como un armisticio, como una simple ausencia de guerra manifiesta. Es éste un concepto pobre, engañoso y manipulable por los poderes establecidos, por los grupos de presión y por los grandes intereses del complejo militar, comercial e industrial de nuestro tiempo. Para una auténtica educación no-violenta y pacificadora hemos de evitar esta interpretación simplista y buscar su significación profunda, aunque el concepto de paz, por ser un concepto elevado, sea un difícil de expresar en palabras, por lo que cualquier definición es solamente aproximada.

Si quieres ver la luz ¿qué haces? Abres la ventana y la percibes directamente, pero es imposible que la describas con exactitud. Igualmente si quieres saber lo que es la paz, debes experimentarla directamente tú mismo, aunque después te sea imposible definirla. Esto no obstante, podemos intentar una definición aproximada, que nos conducirá a considerar la paz como la plenitud, tranquilidad y sosiego de espíritu que nace de la armonía.

Esta armonía, en el ser humano individualmente considerado, es fruto de la progresiva autorrealización personal por el camino del amor universal, de la tolerancia y de la no-violencia, es decir, del estar y del saberse rectamente

${ }^{9}$ Budha: Dhammapada, 10: 1-2. 
orientado hacia su fin último, un fin que está en potencia dentro de todos y cada uno de nosotros mismos.

Colectivamente esta armonía es fruto de la plena realización social, a la cual se llega a través de una convivencia cimentada sobre la verdad, sobre el amor universal, sobre la no-violencia, sobre la justicia, sobre la tolerancia, sobre la compasión y sobre el respeto a los derechos humanos, forma de convivencia que constituye el ambiente idóneo para la plena autorrealización individual de cada uno de los ciudadanos.

En consecuencia el concepto de pacifismo, sobrepasando las interpretaciones más o menos superficiales y más o menos manipuladas en los ambientes políticos, que tratan de compaginarlo con el nacionalismo, el partidismo y el militarismo establecidos, debe ser entendido en el sentido de "defensa, construcción y mantenimiento de la paz y de un orden social justo que respete en su integridad la Declaración Universal de los Derechos Humanos por medios pacíficos y no-violentos ${ }^{9}$. Su alcance ha sido perfilado en el conocido decálogo "Por qué soy pacifista» de Eulogio Díaz del Corral, que nos ofrece las siguientes razones:

1. Porque creo que el derecho a la vida es el derecho fundamental de todos los seres (entre ellos los seres humanos).

2. Porque creo en la bondad natural del ser humano, cuya vida debe desarrollarse con amor y solidaridad, y no con egoísmo y competencia.

3. Porque no odio a nadie ni creo en campañas contra nadie.

4. Porque creo que los pueblos tienen derecho a decidir libremente su futuro.

5. Porque la violencia da la victoria al más fuerte, que independientemente de su fuerza, puede o no tener razón.

6. Porque la guerra se lleva los recursos humanos y económicos que necesitamos para luchar contra la pobreza, la marginación, la incultura y las enfermedades.

7. Porque la guerra la deciden unos -los que mandan- y son otros -los que deben obedecer-quienes tienen que ir a entregar sus vidas.

8. Porque las guerras siempre ocultan intereses económicos de los que ya son poderosos y opresores.

${ }^{9}$ Llorenç VIDAL: Reflexión pacifista después del referéndum, en Última Hora, Palma de Mallorca, 4 de abril de 1986. 
9. Porque las guerras las ganan los estados y siempre las pierden los individuos.

10. Porque los verdaderos héroes de la Humanidad -como Jesucristo, Buda, Lao-Tse, San Francisco de Asís, Ramakrishna, Mahatma Gandhi, Martin Luther King, Madre Teresa de Calcuta, etc.- nos muestran el camino de la no-violencia para reivindicar y defender los derechos humanos y alcanzar la auténtica paz»10.

El tercer puntal -en realidad el básico, ya que los dos anteriores derivan de éste- en el que se apoya el Día Escolar de la No-violencia y la Paz es el concepto de amor universal -omniabarcante, sin reverso de egoísmo y sin contraparte de odio para nadie-, que surge de la idea de unidad de la vida, de unidad de todo lo existente, de unidad del Cosmos, y podemos, a efectos didácticos, definirlo fenomenológicamente como bondad de corazón hacia todos los seres. Es una fuerza dinámica que nace del descubrimiento vital -consciente o inconsciente- de la fraternidad universal, es decir, del hecho de que todos los seres somos hermanos, sin distinción de forma, raza, lengua, nacionalidad, concepción de la vida o manera de ser individual de cada uno.

En consecuencia, el mensaje permanente de la jornada reza:

"Amor universal, No-violencia y Paz.

El Amor universal es mejor que el egoísmo,

la No-violencia es mejor que la violencia

la Paz es mejor que la guerra" 11.

Y en el mensaje profundo del DENIP leemos:

"Busca tu Luz interior y desarrolla la bondad de corazón hacia todos los seres como fuerza de tu vida, de todos tus pensamientos, de todas tus palabras y de todas tus acciones» 12 .

Este concepto de amor universal, que constituye la esencia común de todas las religiones y de todas las filosofías éticas de la vida, puede resumirse en la "Regla de Oro de la Conducta Humana», que dice:

"Ama al prójimo como a ti mismo. No hagas a los demás lo que no quisieras que los demás hicieran contigo. Haz a los demás lo que quisieras que los demás hicieran contigo. Porque todos somos hermanos y porque, más pronto o más tarde, lo que siembres eso mismo recogerás» 13.

10 Eulogio DIAZ del CoRRal: Por qué soy pacifista, en DENIP, Día Escolar de la No-violencia y la Paz, 310 edición, Cádiz, 1994, p. 4.

11 Llorenç VIDAL: Orientaciones básicas del Día Escolar de la No-violencia y la Paz, en Noviolencia y Escuela, p. 39.

12 lbidem, p. 40.

13 Ibidem, p. 39. 
Como dice Helena Petrovna Blavatsky en su obra «La Clave de la Teosofía».:

"Teniendo la humanidad una misma y única esencia, y siendo esa esencia una -infinita, increada y eterna, ya la llamemos Dios o Naturaleza-, nada, por lo tanto, puede afectar a una nación o a un hombre sin afectar a todas las demás naciones y a todos los demás hombres. Tan cierto y obvio es esto, como que una piedra tirada en un estanque pondrá en movimiento pronto o tarde toda gota de agua en él contenida»14.

Y, convencido de este profundo aserto, su discípulo John B.S. Coats afirma que "La fraternidad universal de la humanidad es la verdad fundamental de la existencia» 15 .

Este concepto de amor universal activo comprende otros conceptos derivados del mismo, como el de no-violencia, tolerancia, solidaridad, compasión hacia todos los seres, etc.

Tensin Gyatso, XIV Dalai Lama, en su libro «El arte de la compasión» establece la correspondencia entre el amor como bondad de corazón hacia todos los seres y la compasión como un paralelismo natural, al decir:

«Al igual que la compasión es el deseo de que todos los seres queden libres de sufrimiento, el amor-bondad es el deseo de que todos disfruten de la felicidad» 16.

Y el Coordinador Adjunto del DENIP, en sus «Pensamientos sobre la compasión" precisa que "la compasión es sentir como propio el dolor de los demás seres», actitud que «implica aliviar el sufrimiento y evitarlo si se puede»17.

Ambos, amor y compasión, están en la base dinámica de la no-violencia como forma de vida y como método de acción y de educación.

Sobre estos conceptos se cimientan la Pedagogía de la No-violencia y la Paz y la Educación No-violenta y Pacificadora o Educación en y para la Noviolencia y la Paz, de las que el DENIP es abanderado y constante e incansable propulsor desde 1964 y que -construidas sobre el pensamiento y la acción de algunos grandes educadores, educadores en el sentido más amplio de la palabra, de los tiempos contemporáneos (Mahatma Gandhi, Rabindranath Tagore, María Montessori, Paulus Geheb, Pierre Bovet, Juan XXIII, Martin Luther King, etc.)- durante estos últimos años, como una nueva corriente

14 Helena Petrovna Blavatsky: La Clave de la Teosofía, Edit. Kier, Buenos Aires, 1976, p. 40.

15 John B. S. Coars. Citado en Ideario No-violento, 22, p. 15

16 Tensin Gyatso, XIV Dalai Lama: El arte de la compasión, con la contribución de Nicholas Vreeland, Edit. Grijalbo, Barcelona, 2002, p. 68.

${ }^{17}$ Eulogio DIAZ del CoRral: Pensamientos sobre la compasión, I, Revista cultural Tántalo, $\mathbf{n} 1$. 30. Cádiz, 2003, y en Eulogio Díaz del Corral: Pensamientos, http://es.geocities.com/ahimsadenip/eulogio5.html. 
educativa, se están abriendo paso en la panorámica pluralista de la pedagogía mundial.

No se trata -como algunos podrían pensar, debido al metodologismo actualmente imperante- de una directriz puramente didáctica, sino que se levanta con unas aspiraciones y una proyección mayores, ya que, como consecuencia de su gestación histórica, la Pedagogía de la No-violencia y la Paz se nos presenta como "una inspiración ideal que aspira a informar todo el quehacer educativo de la humanidad y que, en su aspecto teórico, se integra en la Pedagogía General como un capítulo fundamental de la misma y en estrecha relación e interdependencia con la educación religiosa, moral y social»18, ya que, como dice un adagio pacifista,

«Si quieres la paz, no prepares la guerra...

Si quieres la paz, prepara la paz...

Si quieres la paz, edúcate y educa para la paz...».

A pesar de que a menudo en artículos, libros y diccionarios pedagógicos se la presenta superficialmente como un simple corolario educativo de las resoluciones de los organismos internacionales, de las enseñanzas de las iglesias o de las opiniones de los movimientos pacifistas, la Educación No-violenta y Pacificadora tiene una sustantividad propia, que no siempre han sido capaces de captar en toda su profundidad algunos tratadistas demasiado preocupados por los aspectos sociológicos, religiosos o tecnológicos de la educación.

En el marco de las Ciencias de la Educación, la Pedagogía de la No-violencia y la Paz, en su concepción original propagada a partir del DENIP, se define por una aspiración general, unos objetivos, una metodología y unas realizaciones concretas.

- Su aspiración general es incluir entre los objetivos fundamentales de la educación -en todos sus niveles y modalidades- el desarrollo del espíritu de amor universal, no-violencia y paz, con el propósito de contribuir a la formación integral del educando y procurar un mundo más fraterno, menos violento y más pacífico.

- Sus objetivos básicos u objetivos-tendencia, derivados de la aspiración ideal, pueden sintetizarse en las tres tareas siguientes:

Primer objetivo: Conscienciación, es decir, despertar y desenvolver una consciencia humana fraternal, no-violenta y pacífica, que presupone en descubrimiento y la conquista progresiva de la paz interior, el desarme de los corazones y la creación de un sentimiento profundo de hermandad universal;

18 Llorenç VIDAL: Fundamentación de una Pedagogia de la No-violencia y la Paz, Edit. Marfil, Alcoy, 1971, p. 176. 
Segundo objetivo: Ejercitación no-violenta y pacificadora, basada en la humanización progresiva del instinto luchador por obra del amor, ejercitación que debe conducir al desarrollo de hábitos, destrezas y actitudes, y

Tercer objetivo: Responsabilización del ser humano y de la sociedad en la obra individual y colectiva de la pacificación interior, del respeto activo por todas las formas de vida y por los derechos humanos, así como la instauración de una verdadera paz por medio de la no-violencia ${ }^{19}$.

En resumen, la Educación No-violenta y Pacificadora o Educación en y para la No-violencia y la Paz debe ser comprendida como aquella corriente educativa que aspira a despertar y a cultivar las semillas de amor universal, no-violencia y paz que están latentes en el corazón de todos y de cada uno de los seres humanos.

Son innumerables los testimonios que podríamos aportar para avalar la Educación No-violenta y Pacificadora, pero nos limitaremos a los siguientes:

María Montessori, en su conferencia "La Paix et l'Éducation", pronuciada en Ginebra en el año 1932, llamó la atención sobre lo que ella consideraba dos evidencias: la necesidad de crear una ciencia nueva, la ciencia de la paz, y la necesidad de crear una educación nueva, la educación de la paz, ya que, en sus mismas palabras, "es de la paz que depende la vida misma de los pueblos y, tal vez, el progreso o la extinción de toda nuestra civilización”20.

Aldous Huxley, a su vez, elevó su voz para pedir que la educación cumpliera con el fin de "educar a los jóvenes seres humanos para la libertad, la justicia y la paz»21.

En el Preámbulo de la Constitución de la UNESCO leemos:

"Puesto que las guerras nacen en la mente de los hombres (de los seres humanos, mejor) es en la mente de los hombres (de los seres humanos) donde deben edificarse las defensas de la paz»22.

El principio número 10 de la "Declaración de los Derechos del Niño», adoptada y proclamada por la Asamblea General de las Naciones Unidas el 20 de noviembre de 1959, afirma:

19 Llorenç VIDAL: No-violencia y Escuela., pp. 9-11.

20 María Montessori: La paix et l'Éducation, Bureau International d=Education, Genève, 1932, p. 3.

${ }^{21}$ Aldous Huxley. Citado por René Hubert en Traité de Pégagogie Génerale, Presses Universitaires de France, Paris, 1959, p. 4.

22 Constitución de la UNESCO. 
"El niño debe ser protegido contra las prácticas que puedan fomentar la discriminación racial, religiosa o de cualquier otra índole. Debe ser educado en un espíritu de comprensión, tolerancia, amistad entre los pueblos, paz y fraternidad universal, y con plena conciencia de que debe consagrar sus energías y aptitudes al servicio de sus semejantes" 23 .

En el punto octavo del "Programa de Iniciación No-violenta».(1971, versión revisada del 2000), que diseña el plan de acción a largo plazo del Día Escolar de la No-violencia y la Paz, se propugna:

"Fomentar la implantación de una educación en, para y por los derechos y los deberes humanos, la tolerancia, la no-violencia y la paz en las escuelas, colegios, institutos y universidades" 24 .

Y entre las conclusiones de la «Fundamentación de una Pedagogía de la No-violencia y la Paz", publicada en 1971, se reconoce que:

"La Pedagogía de la No-violencia y la Paz-que en cuanto principio general sobrepasa el alcance de todos los sistemas pedagógicos que en ella hasta ahora se han inspirado-puede ser en nuestra época un factor educativo primordial para la preparación de una sociedad futura en la que se supriman o-vitando la tentación de la utopía-se aminoren al máximo los odios, las violencias y las guerras entre los hombres y entre los pueblos, y en la que reinen, de cada día más, la fraternidad, la no-violencia y la paz. Privar de ella a los educandos, invocando una actitud de escepticismo pacifista o pedagógico o en nombre de cualquier teoría más o menos belicista, nacionalista o imperialista, es un fraude a la convivencia actual y venidera de la humanidad»25.

Bosquejada la aspiración general y los tres objetivos básicos de la Educación No-violenta y Pacificadora, podemos preguntarnos: ¿Cuáles son los procedimientos didácticos más adecuados?

En la ya mencionada «Fundamentación de una Pedagogía de la No-violencia y la Paz», a sabiendas de que toda clasificación corre el riesgo de la inexactitud y del esquematismo, propuse una clasificación en siete órdenes:

Procedimientos negativos o de guerra social contra la guerra, entre los cuales se incluyen todos aquellos procedimientos pẹdagógicos extremistas y antimilitaristas, nacidos por reacción contra la éducación belicista, de la que

23 Declaración de los Derechos del Niño, 10.

24 Llorenç VIDAL: Programa de Iniciación No-violenta. Versión revisada del año 2000 en No-violencia, Pensamiento y Espiritualidad, http://es.geocities.com/ahimsadenip/pensamiento.html.

25 Llorenç VIDAL: Fundamentación de una Pedagogía de la No-violencia y la Paz, p. 177. 
inconscientemente toman sus métodos y que, fundamentados en la consigna "guerra y odio a la guerra" y con la pretendida finalidad de suprimir la violencia y la guerra entre los seres humanos y entre las naciones, proponen " $a$ los instintos de lucha del pueblo, a sus sentimientos de odio, un nuevo objetivo, que no es sino el ejército mismo en su institución" 26 . La educadora Mme. Monod protestó ya contra estos sistemas negativos y pidió que "no hagamos de nuestros hijos pacifistas belicosos» 27 . Pierre Bovet, que recoge el anterior testimonio, en su obra "El instinto luchador» concluye:

"Para los verdaderos pacifistas, para aquellos cuyo instinto luchador ha sido verdaderamente sublimado, a la manera de los cuáqueros, una paz internacional perseguida por medio de la guerra social se nos aparece como un bello ideal amalgamado de instintos brutales. Este es el tipo-termina diciendo-de lo que hemos llamado una sublimación fracasada” 28.

Procedimientos preventivos: Desde que la psicología moderna ha puesto de relieve el poder de sugestión y condicionamiento posterior de muchos actos infantiles aparentemente banales y juzgados superficialmente como intrascendentes, y que, sin embargo, pueden servir de adiestramiento prematuro y de acicate ulterior a los instintos latentes en la psique infantil, algunos educadores han pensado en la conveniencia y utilidad de una sana prevención de actitudes negativas.

Se trata de evitar que el niño entre en contacto, prematura e innecesariamente, con hechos, conocimientos y prácticas - castigos violentos en la familia y en la escuela, narraciones bélicas y narraciones infantiles con una dosis tácita o expresa de violencia, instrucción premilitar escolar, etc.- que pueden desviar y pervertir, en vez de ennoblecerlo, el instinto de combate que está dormido en su interior.

Procedimientos lúdicos, que se apoyan en el juego y en el deporte. Superando su dimensionalidad de habituación lúdica negativa, debemos escoger, impulsar y orientar aquellos juegos y deportes que, por su dimensionalidad de habituación lúdica positiva y catártica, puedan ser utilizados como medio para eliminar las tendencias y actitudes violentas y para desarrollar la confianza en sí, la colaboración y el espíritu de juego limpio.

Al mismo tiempo debemos evitar (procurando que nuestra acción no produzca ningún trauma psíquico en el niño) todos aquellos juegos de violencia que conducen a una satisfacción bruta, a una complicación, objetivación y canalización deficiente del instinto de combate, así como el uso de uniformes y juguetes bélicos, que en estos últimos años han inundado, con una sofisticación cada vez mayor, el mercado.

26 Pierre Bovet: El instinto luchador, Francisco Beltrán, Madrid, 1922, p. 335.

27 Wilfred MONOD, en Revue du Christianisme Social, diciembre, 1903.

28 Pierre BOVET: El instinto luchador, p. 336. 
Hermano Pacífico -el protagonista del "Pequeño libro de un solitario"-, al pasar por Biniaïssa, donde vio unos muchachos jugando con espadas y pistolas, dijo:

"Dar a los niños juguetes bélicos es lo mismo que darles prostitutas de cartón para que ejerciten su instinto sexual, ya que el belicismo es a la agresividad humana lo mismo que la prostitución a la sexualidad»29.

Procedimientos de cooperación social, que nos enseñan a conocernos, tolerarnos, dialogar y colaborar los unos con los otros, conscientes de que todos tenemos algo que ofrecer, así como algo con lo que enriquecernos mediante las aportaciones de los demás. Se trata de una serie de procedimientos -introducción de la vida democrática en la escuela, autogobierno de los escolares, asamblea de clase, actividades en equipo, cooperativas escolares, autogestión educativa, dinámica de grupos, etc.- que, si están debidamente enfocados, pueden ayudar a crear una atmósfera que favorezca en todos los miembros de la comunidad educativa la adquisición de actitudes de justicia, diálogo, imparcialidad, autonomía, comprensión, tolerancia, colaboración, respeto, compasión, no-violencia, etc. hacia todos los seres humanos de todas las condiciones.

Este clima convivencial ha de afectar a la escuela en todas sus relaciones de interdependencia y de interinfluencia: en sus relaciones horizontales (profesores entre profesores, alumnos entre alumnos, padres y madres entre padres y madres, etc.) y verticales (dirección, profesorado, alumnado, familias), así como en sus relaciones multidimensionales escuela-entorno, en una actitud comunicativa y dialógica en la cual se eviten y superen las situaciones de violencia, ya que la violencia en las relaciones interpersonales surge como una variedad neurótica del dialogo, en la cual el agresor intenta usar su última argumentación, su sin-razón, de una manera definitiva y contundente. Su fracaso nace precisamente de que la acción humana convivencial no obedece a imposiciones exteriores, sino a un impulso interno y, en consecuencia, se ha de recuperar la situación comunicativa de diálogo si se quiere llegar a un consenso comunitario estable.

Procedimientos de comprensión internacional, que llevan al convencimiento de que todos los seres humanos de todos los países, regiones, razas, lenguas, ideologías y religiones somos hermanos, puesto que la esencia del ser es idéntica para todos. Consecuentemente se ha de llegar a una actitud de respeto y tolerancia hacia todas las formas que tiene de manifestarse esta esencia común en los distintos individuos y en los diferentes colectivos humanos, ya sean étnicos, religiosos, políticos, etc.

${ }^{29}$ Llorenç VIDaL: Pequeño libro de un solitario, 8, versión española sobre el texto de la tercera edición catalana-valenciana-balear publicada por los cuadernos literarios Ponent en 1974, Luis Cárcamo, Editor, Madrid, 1981, p. 25. 
Su culminación está en la conciencia de la fraternidad universal y de la ciudadanía mundial, que tiene una de sus mejores expresiones en aquel pensamiento de Bahá'u'lláh que dice:

"La Tierra es un solo país y la Humanidad sus ciudadanos»30.

La teósofa Annie Besant, en "Barreras infranqueables de la civilización y sus claves", escribió:

«Es necesario que ampliemos la idea de familia hasta el punto de convertir en realidad aquella enseñanza oriental, que consiste en considerar a todo mayor como un padre, a todo coetáneo como un hermano y a todo niño como un hijo, para que de esta manera nuestras relaciones con la sociedad en la que vivimos puedan convertirse en relaciones de amor, de servicio, de ayuda...»31.

Procedimientos de instrucción educativa, que utilizan con una intencionalidad pacificadora distintos recursos tomados bien de la escuela tradicional, bien de la escuela nueva.

Entre los diversos problemas que plantean los procedimientos de instrucción educativa en el campo de la Educación No-violenta y Pacificadora, podemos considerar los siguientes:

Primer problema: Necesidad de una transformación progresiva de la educación escolar y familiar, transformación que ha de afectar a la estructura, organización y orientación del sistema, contenidos, procedimientos y agentes educativos, de acuerdo con la aspiración general y los objetivos de la Educación No-violenta y Pacificadora.

Segundo problema: Enseñanza ocasional y participación activa en conmemoraciones de carácter universalista y pacifista, como el Día de las Naciones Unidas (24 de octubre), Día de los Derechos del Niño (alrededor del 20 de noviembre), Día de los Derechos Humanos (10 de diciembre), Día Escolar de la No-violencia y la Paz (30 de enero), etc., con lecciones ocasionales o con programas específicos de uno o más días, de una semana, de una quincena, etc.

Tercer problema: Partiendo del conocimiento y de la investigación de situaciones experienciales directas o indirectas (testimonios del medio, consulta de documentos escritos, icónicos o audiovisuales, mass media, visitas, entrevistas, cuestionarios sociológicos, etc.), análisis de situaciones conflictivas históricas o actuales -la carrera armamentista y el desarme, por ejemploy búsqueda de las posibles alternativas pacíficas y soluciones no-violentas,

30 BAHÁ=u=lláh. Citado en Ideario No-violento, p. 10.

31 Annie BESANT: Barreras infranqueables de la civilización y sus claves. Citada en Ibidem, p. 16. 
recordando, como afirma Gonzalo Arias, que "siempre existe un camino noviolento, aunque no siempre sea fácil encontrarlo»32.

Cuarto problema: Estudiar en profundidad la Declaración Universal de Derechos Humanos y destacar las figuras históricas y actuales que realmente han trabajado y trabajan pacífica y no-violentamente por la hermandad universal, la no-violencia y la paz, para que se conviertan en arquetipos de vida, evitando la presentación ingenua y no crítica de figuras que creen en la mente y en el sentimiento de los niños y de los jóvenes modelos e ideales erróneos y negativos que ellos inconscientemente tienen la tendencia a imitar.

Procedimientos de despertar interior. Hasta el momento, en los grupos anteriores hemos visto una multiplicidad de procedimientos conducentes a una educación para la paz y la no-violencia, procedimientos buenos en sí, pero parciales e insuficientes, ya que están faltos de una dinámica superior que les proporciones, en su pluralidad, una unidad interior de la que aisladamente carecen. De aquí la necesidad de vitalizarlos por procedimientos de sugestión interior que -a través del contacto personal, el recogimiento, el silencio, la reflexión, la práctica de la compasión y de la acción no-violenta, la meditación pacificadora, etc.- se proponen despertar en el educando el ser íntimo que duerme dentro de nosotros y que se manifiesta como espíritu de amor universal, de no-violencia y de paz, como bondad de corazón hacia todos los seres ${ }^{33}$.

La selección de procedimientos a utilizar para una acción educativa noviolenta y pacificadora, tanto en la escuela como en la familia como en los movimientos de juventud, es un tema de vital importancia, ya que -de acuerdo con el antimaquiavelismo del Mahatma Gandhi- "la definición, por clara que sea, del objetivo que queremos alcanzar, y nuestro deseo de llegar a él, no son suficientes para conducimos al mismo, mientras que no conozcamos o no utilicemos los medios necesarios" 34 .

He ahí el diáfano consejo de Martin Luther King en su libro "El clarín de la conciencian:

«Para alcanzar un fin pacífico, debemos emplear medios pacificos. $Y$ con estas palabras, como análisis final, pretendo decir que el fin y los medios deben estar en estrecha correspondencia, pues el fin ya está contenido en los medios, y, finalmente, unos medios destructivos nunca podrán llevarnos hacia un fin constructivo... Y también

32 Gonzalo ARIAS: La no-violencia, ¿tentación o reto?, Ed. Sígueme, Salamanca, 1977, p. 147.

${ }^{33}$ Llorenç VIDAL: Fundamentación de una Pedagogía de la No-violencia y la Paz, pp. 148-171. Llorenç VIDAL y Eulogio Dfaz DEL CoRRal: Educación para la Convivencia, en Perspectivas Pedagógicas, 41-42, Barcelona, 1978, pp. 221-232.

34 Mahatma GaNDHI, en Amrita Bazar Patrika, 17 de septiembre de 1933, incluido en Extraits $d=$ autres oeuvres en Lettres a $l=A s h r a m$, Éditions Albin Michel, Paris, 1971. 
quiero deciros que, si deseamos la paz en la Tierra y buena voluntad para con los hombres, lo primero que debemos hacer es aceptar la afirmación -contenida en los métodos de la no-violencia-de que toda vida humana es sagrada»35.

Éstos son, vistos sumaria y rápidamente, los siete órdenes de procedimientos básicos, que en el posterior libro "No-violencia y Escuela».se reenfocan funcionalmente para la práctica del DENIP a través de cinco pasos didácticos, lo que constituye una visión complementaria de tipo aplicado...

Y, en este marco filosófico y pedagógico, en parte surgido gracias al mismo, es donde adquiere su verdadero significado el Día Escolar de la No-violencia y la Paz, fundado en 1964 y que, con más de cuatro decenios de continuidad, se nos presenta cada 30 de enero, aniversario del asesinato del Mahatma Gandhi, con un triple propósito:

1. Ser una llamada a cada uno de nosotros para que nos autoeduquemos en y por el amor universal, la no-violencia y la paz, ya que para poder educar a los demás primero hemos de educarnos a nosotros mismos, puesto que el amor, la no-violencia y la paz exteriores, para ser auténticas deben ser fruto del amor, la no-violencia y la paz interiores.

2. Ser una llamada a los educadores de todas las ideologías para que en sus escuelas, institutos y facultades se practique anualmente la jornada y se eduque de una manera continuada y permanente en y por el amor universal, la no-violencia y la paz.

3. Ser una llamada a las familias, a las escuelas y a la sociedad para que, superando viejos prejuicios, se transformen en escuelas de educación en y por el amor universal, la no-violencia y la paz...

El Día Escolar de la No-violencia y la Paz (DENIP) es una iniciativa pionera de Educación No-violenta y Pacificadora en la cual están invitados a participar las escuelas de todos los niveles y de todos los países. Es una actividad práctica que no tiene programaciones oficiales ni normas estructurales de acción, ya que el Día Escolar de la No-violencia y la Paz ${ }^{36}$ es una iniciativa que, manteniendo un pequeño núcleo permanente de orientaciones pedagógicas, permite que cada escuela lo aplique de una manera acomodada a su estilo didáctico propio.

Todos aquellos -amigos o simples simpatizantes- que deseen practicar o promover su práctica, es conveniente que se familiaricen con sus orientacio-

35 Martin LUTHER KiNG: El clarín de la conciencia, Aymà Ed., Barcelona, 1973, pp. 106 y 107.

36 Ver DENIP. No-violencia \& Paz. Non-violence \& Peace, http://es.geocities.com/ahimsadenip y Llorenç Vidal: Artículos literarios y filosóficos, http://es.geocities.com/kirigirisu2002/articulos.html. 
nes básicas, cuyo contenido fundamental -si bien es susceptible de múltiples formas personales y colectivas de aplicación- constituye su razón de ser, el impulso dinámico de su fundación y el móvil para su supervivencia.

En 1966, con motivo de su tercera celebración, Camille Drevet, Secretaria General de la Asociación Amis de Gandhi de París, biógrafa y profunda expositora de la vida y de la obra del Mahatma, calificó al DENIP como «un nuevo hogar de paz" 37 , un hogar que puede ser alumbrado en cada escuela y en el corazón de cada alumno.

El filósofo humanitarista rumano Eugen Relgis, desde su exilio en Uruguay y en su libro "Hojas de mi calendario», calificó el DENIP como «una lucecita de los anhelos de liberación que palpita en el cielo nublado» ${ }^{38}$.

El Dr. Franz Rauhut, catedrático de Filología Románica en la Universidad de Würzburg (Alemania) y uno de los más destacados líderes del movimiento pacifista y de objeción de consciencia alemán, en un artículo publicado en la revista Pazifistische Rundschau (Munich) afirma que «el DENIP debería hacerse una costumbre en todas las escuelas del mundo" 39 .

El Día Escolar de la No-violencia y la Paz, que tiene carácter internacional, se celebra anualmente y de manera creciente en miles de escuelas de España y de otros países.

En su presentación en Bruselas en el Primer Foro Europeo de Educación y Cultura para la Paz, organizado por la Université de Paix de Namur, fundada por Dominique Pire, dije:

"Puesto que el Día Escolar de la No-violencia y la Paz ha demostrado su validez en los miles de centros educativos en los que se ha practicado y puesto que es compatible con todas las ideologías y concepciones pedagógicas que respeten los derechos humanos, nuestra propuesta en este Primer Foro Europeo de Educación y Cultura para la Paz -y lo mismo digo a los lectores desde estas páginasconsiste en sugerir a los movimientos y asociaciones aquí reunidos que, coordinadamente con sus actividades propias, y manteniendo siempre su carácter no estatal, no gubernamental, no oficial, independiente, libre y voluntario, promuevan en los centros educativos de sus respectivos países la práctica anual del Dia Escolar de la No-violencia y la Paz, como punto de partida y de apoyo para una Educación Pacificadora de carácter universal, abierto, profundo y permanente.

37 Camille Drevet: De Cadix à l'Inde, en Bulletin d'Information des Amis de Gandhi, 31/1, Paris, 1966, p. 3.

38 Eugen Relgis: Hojas de mi calendario, Ed. Humanidad, Montevideo, 1970, p. 141.

${ }^{39}$ Franz RaUHUT: Lorenzo Vidal als einen der kenntnisreichten und klüngsten unter Gewaltfreien und Pazifisten von heute, en Pazifistische Rundschau, 11, München, 1975, pp. 18-20. 
"En aquellas escuelas en las que no se practica ningún tipo de Educación para la Paz, el Día Escolar de la No-violencia y la Paz es un punto de partida para una Educación Pacificadora de carácter permanente...

"En aquellas escuelas en las que ya se desarrollan actividades de Educación Pacificadora, el Día Escolar de la No-violencia y la Paz es un punto de apoyo para la continuidad y para la profundización en el mensaje del amor universal, la no-violencia y la paz...

"Y si alguna escuela lo practica dedicándole un día, una semana o una quincena de su tiempo escolar, pero no lo continúa después mediante una Educación Pacificadora de carácter permanente, es, por lo menos, una pequeña semilla que queda depositada en el corazón subconsciente de sus alumnos... Tal vez otro día caiga la lluvia que la haga nacer, crecer y fructificar" 40.

Para facilitar su contextualización en el marco del movimiento disperso, amplio y pluralista de Educación en y para la No-violencia y la Paz, en el año 1971 y anexo a la «Fundamentación de una Pedagogía de la No-violencia y la Paz» 41, publiqué el "Programa de Iniciación No-violenta", que, en versiones revisadas, apareció también en 1981 en el "Ideario No-violento" 42 y en 1985 en "No-violencia y Escuela» 43 . Ahora, ya en la perspectiva de los años 2000 , se ha actualizado nuevamente en una revisión que, en su redacción actual, ofrezco a Vds. como un texto de profundización para la práctica del DENIP. Se trata de un decálogo básico de sugerencias para una participación personal, autoformativa y activa en la obra de la no-violencia. Dice así:

1. Tomar conciencia personal de que la no-violencia-en calidad de instinto de lucha humanizado por el amor, de respeto integral por la vida y como actitud de renunciar a matar y a dañar a los demás seres por medio del pensamiento, la palabra y la acción-es, ante todo, una forma de vida a la que se llega por aceptación interior.

2. Dar cabida al pacifismo no-violento, a la tolerancia de las diversidades y a la idea de ciudadanía mundial en nuestra concepción del mundo y de la existencia.

3. Pedir y apoyar las peticiones para la inscripción de la no-violencia y de la objeción de conciencia en la >Carta Universal de Derechos Humanos=.

40 Llorenç VIdal: Présentation de la Journée Scolaire de la Non-violence et de la Paix, en el Primer Foro Europeo de Educación y Cultura para la Paz, Bruselas, del 15 al 17 de septiembre de 1986. Transcrito en DENIP, 24, Mallorca, 1987, p. 4.

41 Llorenç VIDaL: Fundamentación de una Pedagogía de la No-violencia y la Paz, p. 182.

42 Llorenc VIDAl y Eulogio DIAZ DEL CORRAL: Ideario no-violento.33 preguntas y respuestas sobre la No-violencia y la Paz, p. 25.

43 Llorenç VIDaL: No-violencia y Escuela, pp. 159 - 161. 
4. Humanizar progresivamente nuestro propio instinto luchador para transformarlo de violento en no-violento. Estudiar y ejercitarnos, individualmente o formando grupos de amigos de la no-violencia, en el conocimiento y en la práctica de la filosofía y de la acción no-violenta.

5. Participar activamente, cada uno según su estilo personal, en las acciones en defensa de la vida y en pro de la supresión de la pena de muerte (en todas sus formas); en favor de la tolerancia y del respeto a las minorías, a las individualidades y a los derechos humanos en cualquier parte del globo en que sean violados; contra el hambre en el mundo, contra el racismo, contra la xenofobia, contra el terrorismo, contra la carrera armamentista y en pro del desarme; en actividades de ayuda a los necesitados, oprimidos, marginados, desheredados, inmigrantes, refugiados, presos, etc., con el fin de edificar la paz desde abajo.

6. Difundir, enseñar y propagar el principio de que la no-violencia activa - como fuerza constructora de la paz, de la justicia social, de la tolerancia $y$ de la concordia-debe desplazar progresivamente a la violencia destructora en las relaciones entre los seres humanos y entre los pueblos y entre éstos y la naturaleza.

7. Si llega el caso de promover o participar en alguna reivindicación social, en vez de acudir a la violencia, al terrorismo o al sabotaje, aplicar las técnicas de lucha no-violenta: manifestación no-violenta, no-cooperación o desobediencia civil.

8. Fomentar la implantación de una educación en, para y por los derechos y los deberes humanos, la tolerancia, la no-violencia y la paz en las escuelas, colegios, institutos y universidades.

9. Abogar por la creación de un cuerpo voluntario no-violento de la paz, que realice un servicio cívico sin armas y que sea equivalente, en todo, al servicio militar en los estados donde éste tenga un carácter obligatorio.

10. Utilizar toda nuestra fuerza individual, colectiva y religiosa para transformar progresivamente las actuales estructuras violentas en estructuras no-violentas, que respeten en su integridad la Declaración Universal de Derechos Humanos, seguros que, de esta manera, rendimos un alto servicio a nuestro propio país y a la comunidad internacional ${ }^{44}$.

En mi libro de poemas mallorquines «Estels filants».(«Estrellas fugaces») caractericé la jornada en el siguiente haikai:

44 Llorenç VIDAL: Programa de Iniciación No-violenta (P.I.N.), revisión 2000, en No-violencia, Pensamiento y Espiritualidad (ver URL de la página web en la nota número 24). 


\author{
«Petita flama \\ que encén, cada any que arriba, \\ milers de torxes» 45 . \\ ( Pequeña llama \\ que enciende de año en año \\ miles de antorchas").
}

Que esta pequeña llama del mensaje del Día Escolar de la No-violencia y la Paz y de la Educación No-violenta y Pacificadora ilumine durante todo el nuevo milenio la intimidad de nuestros corazones, de nuestras escuelas, de nuestro hacer pedagógico diario y de todas nuestras comunidades de educación y de convivencia.

Nuestros ideales, sin duda, son elevados y nuestras metas tal vez lejanas, pero, como escribió Albert Schweitzer:

"Los ideales se parecen a las estrellas en que nunca los alcanzamos, pero como los navegantes dirigimos por ellos el rumbo de nuestras vidas» 46 .

\title{
Anexo
}

\section{Día Escolar de la No-violencia y la Paz (DENIP)}

\section{Orientaciones básicas}

\author{
(30 de enero) \\ (Calendarios escolares del Hemisferio Sur: 30 de marzo)
}

El Día Escolar de la No-violencia y la Paz (DENIP), fundado en 1964, es una iniciativa pionera de Educación No-violenta y Pacificadora en la que están invitados a participar los centros educativos de todos los niveles. Es una actividad práctica que no tiene programaciones oficiales ni líneas estructurales de acción, como suelen tener los movimientos organizados burocrática o políticamente, ya que el DENIP es simplemente un mensaje impulsor de la Educación No-violenta y Pacificadora, un mensaje que, manteniendo un pequeño núcleo permanente de orientaciones básicas, permite la libre aplicación en cada centro educativo según su estilo didáctico propio.

45 Llorenç VIDaL: Estels filants, Nova Arcàdia, Mallorca - Cádiz, 1991, p. 19.

46 Albert SCHWEITzER. Citado en José Rfos: Albert Schweitzer y su veneración por la vida, Montevideo, 1967, p. 51. Ver también Llorenç VIDAL: Pour inaccessible qu'il paraisse, l'idéal est un guide, en la obra colectiva que recoge las aportaciones al Foro para la Paz titulado La guerre ou la paix?, París, UNESCO, 1980, pp. 204-206. 
Las orientaciones básicas a las que acabamos de referirnos son las siguientes:

1. El Día Escolar de la No-violencia y la Paz es una llamada a los educadores de buena voluntad de todas las ideologías para que tomen conciencia de que uno de los objetivos fundamentales de la educación consiste en despertar y desenvolver en los educandos el espíritu de amor universal, no-violencia y paz, con el propósito de cooperar al desarrollo de su personalidad integral y procurar un mundo más fraterno, más respetuoso con los derechos humanos, menos violento y más pacífico.

2. El DENIP se celebra el 30 de enero (o uno de los días próximos, si el 30 es jornada no lectiva) de cada año, en el aniversario del martirio del Mahatma Gandhi, el gran apóstol de la no-violencia en el mundo actual. En aquellos países en los que el 30 de enero coincide con periodo de vacaciones, puede conmemorarse el 30 de marzo (o uno de los días próximos si el 30 es no lectivo).

3. Como resumen didáctico del pensamiento y de la práctica pacificadora y no-violenta de los grandes líderes de este movimiento -M. Gandhi, M. L. King, Lanza del Vasto, etc.- el mensaje básico del DENIP dice:

Amor universal, No-violencia y Paz.

El Amor universal es mejor que el egoísmo,

la No-violencia es mejor que la violencia

y la Paz es mejor que la guerra.

4. El procedimiento didáctico ha de ser vivencial y -dentro de una gran libertad de opciones y alternativas- puede consistir en:

4.1. Motivación.

4.2. Desarrollo (breve esbozo biográfico del Mahatma Gandhi, Martin Luther King o de algún otro pacifista no-violento; investigación, análisis y estudio de alguna situación conflictiva y/o resolución no-violenta de conflictos, etc.).

4.3. Como corolario surgido del desarrollo anterior, comentario del mensaje básico del DENIP.

4.4. Breve meditación pacificadora, silencio o reflexión personal sobre el mensaje básico del DENIP.

4.5. Actividades expresivas y/o aplicadas de orden actitudinal, práctico, verbal, plástico, dinámico, etc.

5. El DENIP tiene como base la práctica de la fraternidad universal, a través de la Regla de Oro de la Conducta Humana, que, bajo distintas formas, ha sido enunciada por los profetas religiosos de todos los tiempos y culturas, así como por todas las filosofías éticas de la vida, y que dice: 
"Ama al prójimo como a ti mismo. No hagas a los demás lo que no quisieras que los demás hicieran contigo. Haz a los demás lo que quisieras que los demás hicieran contigo. Ya que todos somos hermanos y porque, más pronto o más tarde, lo que siembres eso mismo recogerás».

6. Amigos del DENIP y de la Educación No-violenta y Pacificadora son aquellas personas (adultos, jóvenes, adolescentes y niños) que aceptando la supremacía espiritual, individual y social del amor universal sobre el egoísmo, de la no-violencia sobre la violencia y de la paz sobre la guerra, se esfuerzan, día a día, en vivir y enseñar -por el ejemplo más que por las prédica- los principios inspiradores de la jornada. Se pide a los amigos y simpatizantes que con asiduidad y al menos el 30 de enero dediquen unos minutos a meditar o reflexionar en silencio sobre el mensaje del DENIP.

7. Para quienes deseen desarrollar más plenamente sus potencialidades internas, el DENIP ofrece este mensaje profundo:

Busca tu Luz interior y cultiva la Bondad de corazón hacia todos los seres (es decir, el espíritu de amor universal, no-violencia y paz) como fuerza de tu vida: de todos tus pensamientos, de todas tus palabras y de todas tus acciones.

8. Aunque el DENIP se celebre en una fecha determinada -su práctica puede comprender una o varias jornadas, una semana, una quincena o un periodo indeterminado de tiempo-, su mensaje debe durar todos los días, debe informar toda nuestra educación y debe guiar toda nuestra vida a través de nuestros pensamientos, de nuestras palabras y de nuestras acciones, porque, asumido libremente según el estilo de vida personal de cada uno, puede ser un medio para ayudar al ser humano a liberarse de esclavitudes egoístas (ambientales, corporales y mentales) y un camino para su progresiva autorrealización en un horizonte de fraternidad universal. Por esta razón, el mensaje del DENIP es una semilla que debería llegar a los centros educativos de todo el mundo, para constituir el germen y el punto de apoyo de una educación no-violenta y pacificadora de carácter universal, independiente, abierto, profundo y permanente.

9. Educadores: si tenéis conciencia de la importancia de vuestra labor educativa, ¡haced una pausa en el camino y, en la sencillez de vuestra escuela, practicad anualmente con vuestros alumnos el Día Escolar de la No-violencia y la Paz! Es una buena semilla que cultiváis en su corazón. 
Todos aquellos -amigos o simples simpatizantes- que deseen practicar o promover la práctica del DENIP, es conveniente que se familiaricen con estas orientaciones básicas, cuyo mesaje fundamental, que es susceptible de múltiples formas personales o colectivas de aplicación, constituye su razón de ser, el impulso dinámico de su fundación y el móvil para su supervivencia.

\section{RESUMEN}

El Día Escolar de la No-violencia y la Paz (DENIP), que se practica el 30 de enero, aniversario del martirio del Mahatma Gandhi (en los paises con calendarios del hemisferio sur el 30 de marzo o alguno de los dias próximos), fue fundado en 1964 y es una de las más importantes experiencias de Educación No-violenta y Pacificadora de nuestro tiempo, así como un núcleo en el que se han inspirado y tienen su origen la mayoria de iniciativas actuales de Educación en y para la No-violencia y la Paz.

El DENIP tiene sus fundamentos teóricos en la filosofía y la práctica de la no-violencia (entendida como actitud de renunciar a matar y a dañar a los demás seres por medio del pensamiento, la palabra y la acción, como respeto por la vida y como identificación con todo lo que vive), en la idea de paz positiva y de pacifismo no-violento integral, en el concepto de amor universal y en una concepción original de la Pedagogía y Educación en y para la Noviolencia y la Paz, que se desarrolla a través de tres objetivos básicos (conscienciación, ejercitación y responsabilización del educando) y un haz diversificado de siete órdenes de procedimientos didácticos.

El Dr. Franz Rauhut, Profesor del la Universidad de Würzburg, escribió que el DENIP deberia hacerse una costumbre en todas las escuelas del mundo. Sigue al artículo un anexo con las Orientaciones básicas del Día Escolar de la No-violencia y la Paz concebido como germen, punto de partida y de apoyo para una Educación No-violenta y Pacificadora de carácter universal, independiente, abierto, profundo y permanente.

Palabras clave: Educación, pedagogía, cultura, no-violencia, paz, Mahatma Gandhi, DENIP, día escolar, conscienciación, ejercitación, responsabilización.

\section{ABSTRACT}

The School Day of Non-Violence and Peace (Dia Escolar de la Noviolencia y la Paz, or DENIP), which is observed on January 30, the anniversary of Mahatma Gandhi's martyrdom (in the countries following the southern hemisphere's calendar, it is observed on or about March 30), was founded in 1964. 
It is one of the most important experiences of our times in non-violent and pacifying education and a core event that has inspired and served as origin to the majority of today's initiatives in education in and for non-violence and peace.

The basic theories behind DENIP come from the philosophy and practice of non-violence (which is understood as forbearance to kill and hurt other living beings by thought, word and action, as respect for life and as identification with all living things), in the idea of positive peace and comprehensive non-violent pacifism, in the concept of universal love and in an original conception of pedagogy and education in and for non-violence and peace, which is implemented through three basic objectives (awareness-raising, exercising and making the pupil feel his or her responsibility) and a diversified sheaf of teaching procedures that can be divided into seven orders.

Dr. Franz Rauhut, professor at the University of Würzburg, wrote, "DENIP should be made a custom in all schools throughout the world".. The article is followed by an appendix, "Basic Orientations for the School Day of non-Violence and Peace», which is conceived as the germ, starting point and point of support for non-violent, pacifying education of a universal, independent, open, deep and ongoing nature.

Key words: Education, pedagogy, culture, non-violence, peace, Mahatma Gandhi, DENIP, school day, awareness-raising, exercising, making one fell one's responsibility. 